\title{
Mechanical Systems with Hyperbolic Chaotic Attractors Based on Froude Pendulums
}

\author{
S. P. Kuznetsov, V.P. Kruglov, Yu. V. Sedova
}

We discuss two mechanical systems with hyperbolic chaotic attractors of Smale-Williams type. Both models are based on Froude pendulums. The first system is composed of two coupled Froude pendulums with alternating periodic braking. The second system is Froude pendulum with time-delayed feedback and periodic braking. We demonstrate by means of numerical simulations that the proposed models have chaotic attractors of Smale-Williams type. We specify regions of parameter values at which the dynamics corresponds to the SmaleWilliams solenoid. We check numerically the hyperbolicity of the attractors.

Keywords: hyperbolic chaotic attractors, Smale-Williams solenoid, Bernoulli map.

Received June 14, 2019

Accepted September 09, 2019

S.P. Kuznetsov and V.P. Kruglov acknowledge support from the Russian Science Foundation, grant No. 15-12-20035 (Sections 1-3). Yu. V. Sedova acknowledges support from the Russian Science Foundation, grant No. 17-12-01008 (Section 4).

Sergey P. Kuznetsov

spkuz@yandex.ru

Udmurt State University,

ul. Universitetskaya 1, Izhevsk, 426034 Russia

Saratov Branch of Kotel'nikov's Institute of Radio-Engineering and Electronics of RAS

ul. Zelenaya 38, Saratov, 410019 Russia

Vyacheslav P. Kruglov

kruglovyacheslav@gmail.com

Saratov Branch of Kotel'nikov's Institute of Radio-Engineering and Electronics of RAS

ul. Zelenaya 38, Saratov, 410019 Russia

Yuri Gagarin State Technical University

ul. Politechnicheskaya 77, Saratov, 410054 Russia

Yuliya V. Sedova

sedovayv@yandex.ru

Saratov Branch of Kotel'nikov's Institute of Radio-Engineering and Electronics of RAS

ul. Zelenaya 38, Saratov, 410019 Russia 


\section{Introduction}

Uniformly hyperbolic attractors [1] are genuine chaotic and consist only of saddle trajectories. At every point of a uniformly hyperbolic attractor its tangent space can be decomposed into a direct sum of two subspaces, stable and unstable. It is an important part of the definition that all possible angles between any vector from stable subspace and any vector from unstable subspace are distanced from zero at every point of the attractor. Uniformly hyperbolic attractors are structurally stable. This means that they occupy an open set in the parameter space [1].

The Smale-Williams solenoid [2,3] is a well-known example of a uniformly hyperbolic chaotic attractor. It appears in the phase space of a discrete dynamical system if a torus-form domain undergoes in one discrete time step an $M$-fold longitudinal stretching ( $M \geqslant 2$ is an integer), strong transverse compression and folding in a loop located inside the initial torus. With each repetition of the transformation, the number of curls increases by factor $M$ and in the limit tends to infinity, resulting in a solenoid with a Cantor-like transverse structure. The chaotic nature of the dynamics is determined by the fact that the transformation of the angular coordinate in this setup corresponds to an expanding circle map, or the Bernoulli map $\phi_{n+1}=M \phi_{n}(\bmod 2 \pi)$.

Among possible examples of hyperbolic chaos in systems of various nature we outline mechanical models as they are easily perceived and interpreted in a frame of our everyday experience. We propose to consider two mechanical systems $[4,5]$ with the basic element being a self-oscillating Froude pendulum. The first model is composed of two Froude pendulums on a common shaft interacting by friction [4]. Pendulums are alternately braked by periodic application of external frictional forces. The Smale-Williams solenoid occurs as an attractor of the Poincaré stroboscopic map as we properly specify the system parameters. The second model is composed of only one Froude pendulum interacting with the mechanical time-delay line [5]. The pendulum undergoes periodic braking. The Smale-Williams solenoid in this model appears in the phase space of infinite dimension of the system with time-delay.

We start with a quick overview of the Froude pendulum.

\section{Froude pendulum}

Froude pendulum is a well-known example of a mechanical self-oscillator. It is a weight on a rod of negligible mass. The rod is attached to a sleeve placed on a shaft rotating at a constant angular velocity. In the dimensionless form the governing equation of the Froude pendulum reads

$$
\ddot{x}-\left(a-b \dot{x}^{2}\right) \dot{x}+\sin x=\mu,
$$

where $x$ is an angle of the pendulum displacement from the vertical line. The friction torque between the shaft and the sleeve gives rise to self-oscillatory motions of the Froude pendulum at a certain range of parameters.

If $a>0$, then self-oscillations arise in the system. On the phase plane in Fig. 1a the self-oscillatory modes are represented by limit cycles around the equilibrium state $O$ at $(x, \dot{x})=$ $=(\arcsin \mu, 0)$. For $a$ near zero and small $\mu$, the frequency of the self-oscillations is close to the natural frequency of the pendulum $f=(2 \pi)^{-1}$. With growth of $a$, the limit cycles increase in size, and the frequency $f$ decreases. This is due to the fact that the pendulum approaches the saddle point $S,(x, \dot{x})=(\pi-\arcsin \mu, 0)$, corresponding to a position pointing upwards, where the motion slows down. Further growth of the parameter $a$ leads to a change of the oscillatory movements of the pendulum to periodic rotational motions, which correspond to the limit cycles 

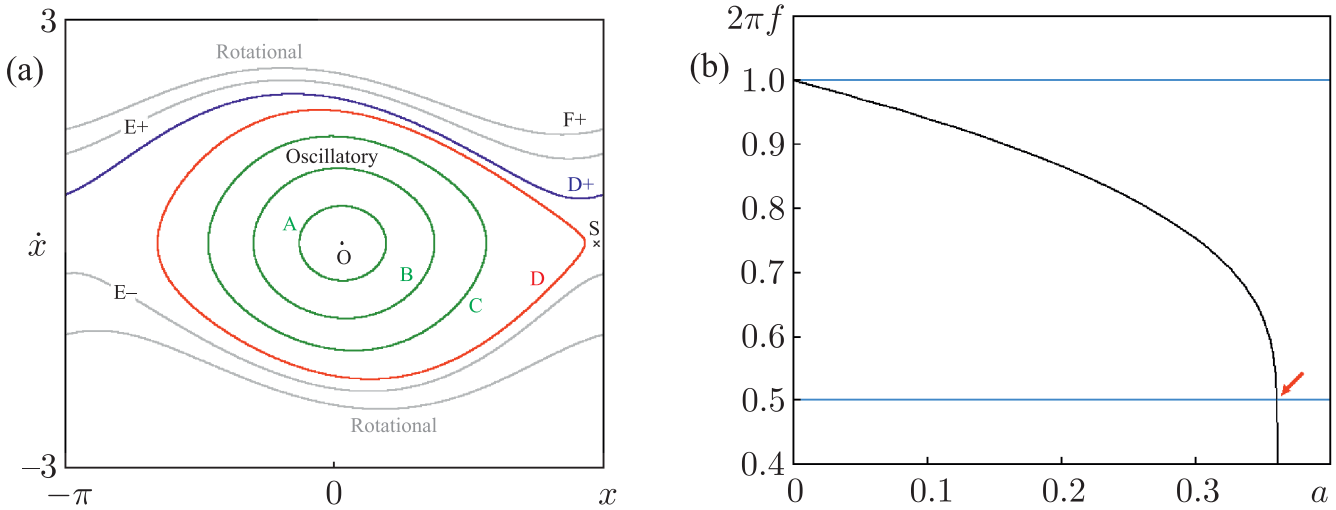

Fig. 1. (a) Attracting limit cycles for various parameters corresponding to sustained periodic motions of a single Froude pendulum: oscillatory A, B, C, D, respectively, at $a=0.03,0.12,0.24,0.36$ and rotational $\mathrm{D}+, \mathrm{E} \pm, \mathrm{F} \pm$ at $a=0.36,0.48,0.6$. (b) The dependence of the frequency of the self-oscillating regimes on the value of $a$. The other parameters are $b=0.16, \mu=0.087$. Panel (a) shows points that are equilibrium states: unstable focus $O$ and saddle $S$. On the panel (b) the arrow indicates the situation where the frequency of self-oscillations is half the frequency of small oscillations of the pendulum.

going around the phase cylinder. The dependence of the frequency of the self-oscillating regimes on the value of $a$ is shown in Fig. 1b.

For further consideration, we select parameters to obtain self-oscillations with the frequency exactly equal to half the frequency of small self-oscillations at $a$ close to zero. At $\mu=0.087$ and $b=0.16$ this is the case if we set $a=0.36$. Then the oscillatory process has an essential second harmonic of the fundamental frequency (this is due to the lack of symmetry of equation (2.1) with respect to the substitution $x \rightarrow-x$ ). If the generated signal acts on a linear oscillator of natural frequency $\omega=1$, one can observe its resonant excitation under the second harmonic of the self-oscillating system.

\section{System of two Froude pendulums with alternating periodic braking}

Let us consider two identical Froude pendulums placed on a common shaft and weakly connected with each other by viscous friction, so that the torque of the frictional force is proportional to the relative angular velocity. Let the motion of pendulums be decelerated alternately by attaching a brake shoe providing suppression of the self-oscillations due to the incorporated sufficiently strong viscous friction. Denoting the angular coordinate of the first and the second pendulum as $x$ and $y$, and the angular velocities as $u$ and $v$, we write down the equations

$$
\begin{aligned}
& \dot{x}=u, \\
& \dot{u}=\left[a-d(t)-b u^{2}\right] u-\sin x+\mu+\varepsilon(v-u), \\
& \dot{y}=v, \\
& \dot{v}=\left[a-d(t+T / 2)-b v^{2}\right] v-\sin y+\mu+\varepsilon(u-v), \\
& d(t)=\left\{\begin{array}{l}
0, t<T_{0}, \\
D, T_{0}<t<T / 2, \quad d(t+T)=d(t) . \\
0, T / 2<t<T .
\end{array}\right.
\end{aligned}
$$


The parameters are assigned as follows:

$a=0.36, \quad b=0.16, \quad \mu=0.087, \quad \varepsilon=0.0003, \quad D=0.8, \quad T=250, \quad T_{0}=T / 4$.

To explain the operation of the system (3.1), we start with the situation where one pendulum is self-oscillating, and the second is suppressed to small oscillations by braking. Due to the fact that the parameters are chosen in accordance with the reasoning of the previous section, the basic frequency of the self-oscillatory mode is half that of the second pendulum. Therefore, when the brake shoe is removed from the second pendulum, it will begin to swing in a resonant manner due to the action of the second harmonic from the first pendulum, and the phase of the oscillations that arise will correspond to the doubled phase of the oscillations of the first pendulum. As a result, when the second pendulum approaches the sustained self-oscillatory state, its phase appears to be doubled in comparison with the initial phase of the first pendulum. Further, the first pendulum undergoes braking, and at the end of this stage, its oscillations will be stimulated in turn by the action of the second harmonic from the second pendulum, and so on.

Since the system (3.1) is nonautonomous, one can go on to discrete time dynamics by constructing the Poincaré stroboscopic map. In our case, taking into account the symmetry of the system in respect to substitution $x \leftrightarrow y, u \leftrightarrow v, t \leftrightarrow t+T / 2$, it is appropriate to use the mapping in half a period of modulation, determining the state vector at the instants of time as

$$
\mathbf{X}_{n}=\left\{\begin{array}{l}
\left(x\left(t_{n}\right), u\left(t_{n}\right), y\left(t_{n}\right), v\left(t_{n}\right)\right), \text { if } n \text { is odd } \\
\left(y\left(t_{n}\right), v\left(t_{n}\right), x\left(t_{n}\right), u\left(t_{n}\right)\right), \text { if } n \text { is even }
\end{array}\right.
$$

The Poincaré map for the vector $\mathbf{X}_{n}=\left(x_{1}, x_{2}, x_{3}, x_{4}\right)_{n}$ is

$$
\mathbf{X}_{n+1}=\mathbf{F}_{T / 2}\left(\mathbf{X}_{n}\right) \text {. }
$$

Since each new stage of the excitation transfer to one or another pendulum is accompanied by a doubling of the phase of oscillations, this corresponds to the Bernoulli map for the phase. If a volume contraction takes place along the remaining directions in the state space of the system, this will correspond to occurrence of the Smale-Williams solenoid as an attractor of the Poincaré map (3.4).

At the stage of the high-amplitude oscillations their waveform differs significantly from the sinusoidal. In this case, evaluation of the phase through the ratio of the variable and its derivative as arctangent is not so satisfactory. We define the phase using a value of the time shift of the waveform with respect to a given reference point, normalized to the characteristic period of the self-oscillatory mode. Let $t$ be a fixed time instant at the activity stage of one of the pendulums, and let $t_{1}, t_{2}$ be the preceding moments of the sign change of the angular velocity from plus to minus, and $t_{2}>t_{1}$. Then we can define the phase as a variable belonging to the interval $[0,1]$ by the relation $\varphi=\left(t-t_{2}\right)\left(t_{2}-t_{1}\right)^{-1}$.

Figure 2a shows a diagram for the phases determined at the end parts of successive stages of excitation of the first and second pendulums, obtained in numerical calculations for a sufficiently large number of the modulation periods. As can be seen, the mapping for the phase in the topological sense looks equivalent to the Bernoulli map $\varphi_{n+1}=2 \varphi_{n}+$ const $(\bmod 1)$. Indeed, one complete round for the preimage $\varphi_{n}$ (i.e., a unit shift) corresponds to a double round for the image $\varphi_{n+1}$.

Figure $2 \mathrm{~b}$ shows an attractor of the Poincaré stroboscopic map. Although visually the object looks like a closed curve, in fact it has a fine transverse structure, the visualization of which requires high-accuracy calculations, and evolution in discrete time corresponds to jumps of the representing point around the loop accordingly to iterations of the Bernoulli map. 

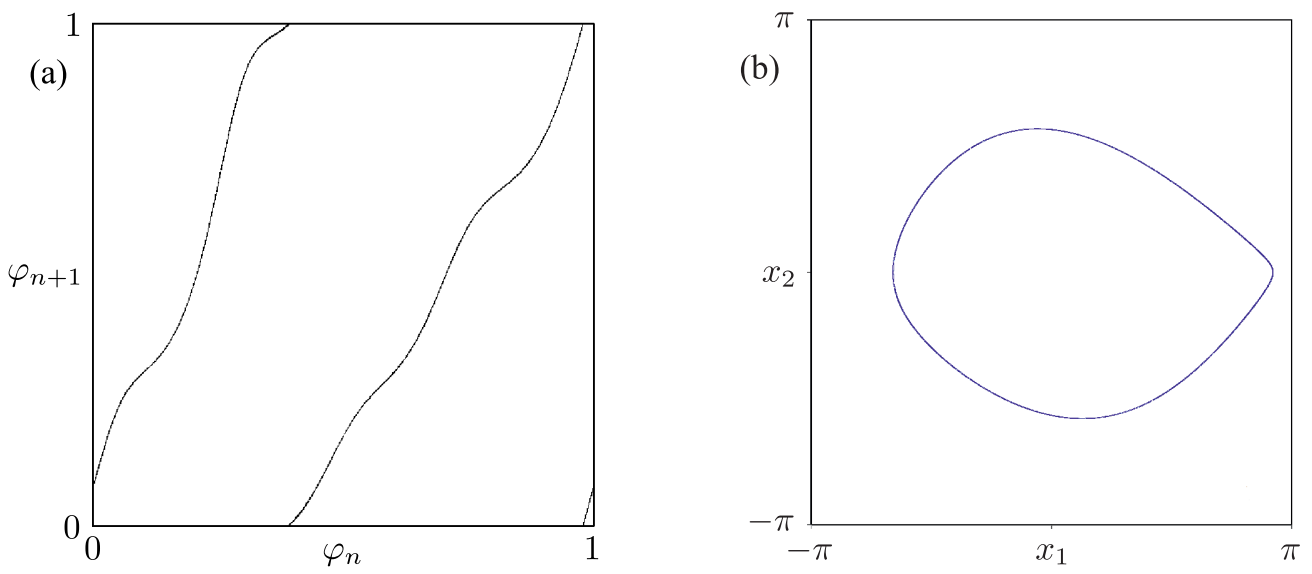

Fig. 2. (a) A diagram illustrating the transformation of the phases of pendulums in successive stages of activity every half a period of modulation. (b) Attractor of the Poincaré stroboscopic map, which is a Smale-Williams solenoid in projection onto the plane of two variables.

At the parameters assigned according to (3.2), the Lyapunov exponents for the Poincaré map attractor are

$$
\Lambda_{1}=0.65 \pm 0.007, \quad \Lambda_{2}=-5.89 \pm 0.02, \quad \Lambda_{3}=-9.46 \pm 0.02, \quad \Lambda_{4}=-20.73 \pm 0.02 .
$$

The presence of a positive exponent $\Lambda_{1}$ indicates a chaotic nature of the dynamics. Its value is close to $\ln 2=0.693 \ldots$, which agrees with the approximate description of the evolution of the phase variable $\varphi$ by the Bernoulli map. The action of the Poincaré map $\mathbf{F}$ in the fourdimensional space is accompanied by stretching in the direction corresponding to the phase $\varphi$ and contracting along the remaining three directions. This corresponds to the Smale - Williams construction, namely, in the four-dimensional space.

Figure 3a shows a chart of regimes on the parameter plane $(\varepsilon, D)$, i.e., of the coupling parameter versus the dissipation parameter introduced by the brake pad during the braking

(a)

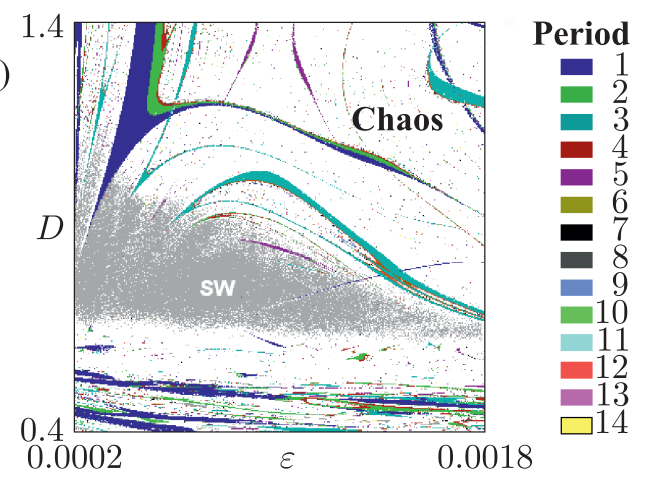

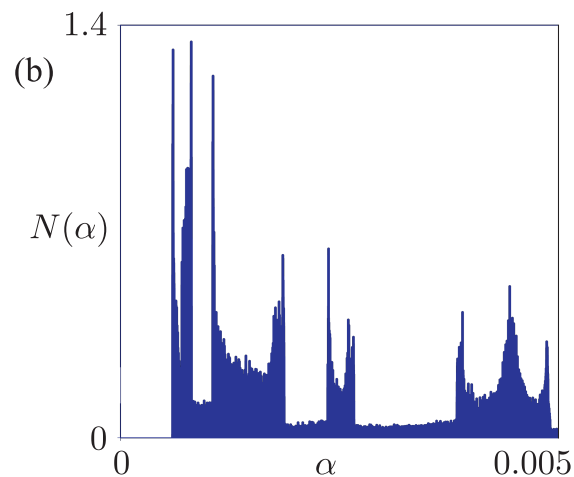

Fig. 3. (a) Chart of regimes of the system (3.1) on the plane $(\varepsilon, D)$, where areas of chaos and of periodic motions are shown. The region of hyperbolic chaos associated with the Smale-Williams attractor is shown in gray and marked as SW, other chaotic regimes are shown in white. The legend for periodic regimes is shown on the right. The periods indicated in colors are measured in units of modulation period. The fixed parameters are $a=0.36, b=0.16, \mu=0.087, T=250, T_{0}=T / 4$. (b) Histogram of the angles of intersection of stable and unstable subspaces for the hyperbolic attractor of the Poincaré map of the system (3.1). $a=0.36, b=0.16, \mu=0.087, \varepsilon=0.0003, D=0.8, T=250, T_{0}=T / 4$. 
stages. The remaining parameters correspond to the situation where the period of relaxation selfoscillations at the activity stage is exactly twice the period of small oscillations. The structure of the regions is determined by the excitation transfer from one stage of activity to the next one in the course of the system operation. In the central part of the chart one can see a broad area SW, where hyperbolic chaos takes place. To identify the Smale-Williams solenoid, the topological equivalence of the map for the phase to the Bernoulli map was tested visually or automatically, using a specially developed algorithm. The exit of this area down corresponds to the fact that dissipation at the stages of breaking decreases and becomes too small to provide a sufficient degree of damping of the natural oscillations of the pendulum after the previous activity stage, which makes a competing contribution to the stimulation of the oscillatory process at the new activity stage, so that the phase doubling transfer mechanism is violated. The exit through the upper boundary of the SW region corresponds to the fact that at the curve representing the graph for the phases, a bend is formed on one of the branches, and then a maximum and a minimum appear, so that the monotony property is lost. This means that when a stretched double loop is inserted into the original toroidal region in the solenoid construction procedure, there appears a local fold on the loop, which leads to disruption of the proper solenoid structure. When the parameters at the upper edge of the region SW are varied, periodic motions become possible; on the chart one can see a set of periodicity tongues. Visually, they look similar to the classical synchronization Arnold tongues, but the fundamental difference from the classical picture is that between them we have chaotic dynamics rather than quasi-periodicity.

Figure $3 \mathrm{~b}$ shows a histogram of the angles of intersection of stable and unstable subspaces for a trajectory on the attractor of the Poincaré map of the system with parameters assigned according to (3.2). The fact that the distribution is separated from zero confirms the hyperbolic nature of the attractor. Similar results are obtained for parameters of the system in a certain range, which corresponds to the structural stability inherent in the hyperbolic attractor. The technique of the performed numerical test was developed in [6].

\section{Froude pendulum with delayed feedback and periodic braking}

Let us consider a Froude pendulum placed on a rotating shaft and contacting with a mechanical time-delay transmission line (a spring with a free end). Let the motion of the pendulum be decelerated periodically by attaching a brake shoe. Denoting the angular coordinate of the pendulum as $x$, and the angular velocity as $u$, we write down the equations

$$
\begin{aligned}
& \dot{x}=u, \\
& \dot{u}=\left[a-d(t)-b u^{2}\right] u-\sin x+\mu+\varepsilon(u(t-\tau)-u(t)), \\
& d(t)=\left\{\begin{array}{l}
0, t<T_{0}, \\
D, T_{0}<t<T / 2, \quad d(t+T)=d(t) . \\
0, T / 2<t<T .
\end{array}\right.
\end{aligned}
$$

The system (4.1) is nonautonomous, with periodic parameter modulation, so we can use the description of the dynamics in terms of discrete time by means of the stroboscopic Poincaré map

$$
\mathbf{X}_{\mathrm{n}}=\mathbf{F}_{\mathrm{T}}\left(\mathbf{X}_{\mathrm{n}-1}\right)
$$


Here the vectors $\mathbf{X}_{\mathrm{n}}$ denote the sets of quantities $x\left(t_{n}\right), \dot{x}\left(t_{n}\right)$ together with functions $\dot{x}(t-\tau)$, $t \in\left[t_{n}-\tau, t_{n}\right)$ determining the state of the system at the time instants $t_{n}=n T$, and should be interpreted as elements of infinite-dimensional state space.

Parameters are assigned as follows:

$$
\begin{gathered}
a=0.36, \quad b=0.16, \quad \mu=0.087, \quad \varepsilon=0.0003, \\
D=0.8, \quad T=250, \quad T_{0}=T / 4, \quad \tau=T / 2 .
\end{gathered}
$$

Let us start with a situation where the braking is not applied, and the pendulum performs relaxation self-oscillations, in which, due to the selection of the parameters, the frequency is half that for the small oscillations of the pendulum. The signal generated at this stage is sent to the time-delay feedback transmission line. Further, the pendulum oscillations are suppressed by application of the brake pad. When braking stops, a new stage of the buildup of the oscillations begins, starting from the practically unexcited state of the pendulum. As we properly select the delay time, the oscillatory process will be stimulated by the resonant action of the second harmonic of the signal received through the feedback circuit and emitted just on the previous stage of intense oscillations. Therefore, the phase of the developing oscillations corresponds to the doubled phase of the main component of oscillations at the prior activity stage. As a result, when the newly arisen oscillations of the pendulum approach the sustained relaxation self-oscillations, their phase will be doubled compared to the phase at the preceding activity stage. Further, the process is repeated again and again. A full cycle corresponding to the modulation period $T$ is accompanied by multiplying the initial phase of the oscillatory process by a factor of 2, i.e., for it, a doubly expanding circle map takes place. Due to compression in the remaining directions in the state space of the map (4.2), the Smale-Williams attractor arises.

Numerical simulations were undertaken. It was demonstrated that the dynamics of phases indeed is described by a map topologically equivalent to the Bernoulli map. The calculation of the largest three Lyapunov exponents of the Poincare map for the attractor at the given parameters (4.3) yields

$$
\Lambda_{1}=0.65, \quad \Lambda_{2}=-6.325, \quad \Lambda_{3}=-7.061
$$

The positive value of the first exponent indicates a chaotic nature of the dynamics, and, as we can see, it is quite close to the value $\ln 2=0.693 \ldots$ (Lyapunov exponent of the Bernoulli map). Other Lyapunov exponents are negative. This corresponds to the Smale-Williams solenoid embedded in the infinite-dimensional space.

Figure 4a shows a chart of regimes on the parameter plane $(\varepsilon, D)$, that is, the time-delay feedback intensity parameter versus the dissipation parameter introduced by the brake pad during the braking stages. One can see a broad domain SW of hyperbolic chaos. Observe a remarkable similarity of the chart to that in Fig. 3; the difference is distinct coloring of the periodicity areas, as in the system of coupled pendulums the phase transfer happens twice on the modulation period, while in the time-delay system it takes place one time on a modulation period. Figure $4 \mathrm{~b}$ shows a histogram of the angles of intersection of stable and unstable subspaces for a trajectory on the attractor of the Poincaré map of the system (4.1) with parameters assigned according to (4.3). The distribution is distanced from zero, which confirms the hyperbolicity of the attractor. The special method of numerical test of hyperbolicity for time-delayed systems was developed in [7]. 
(a)

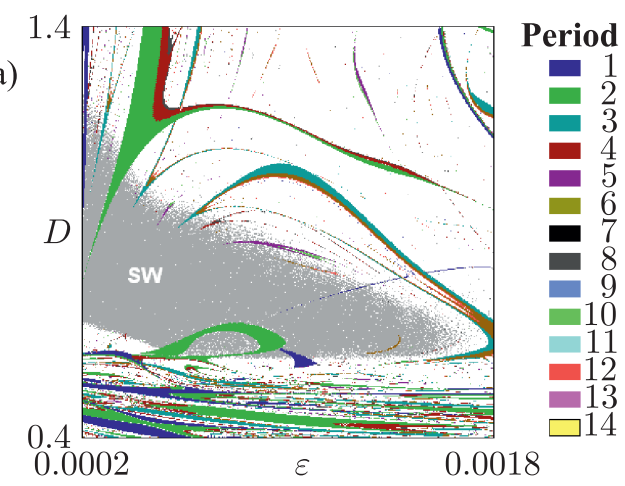

(b)

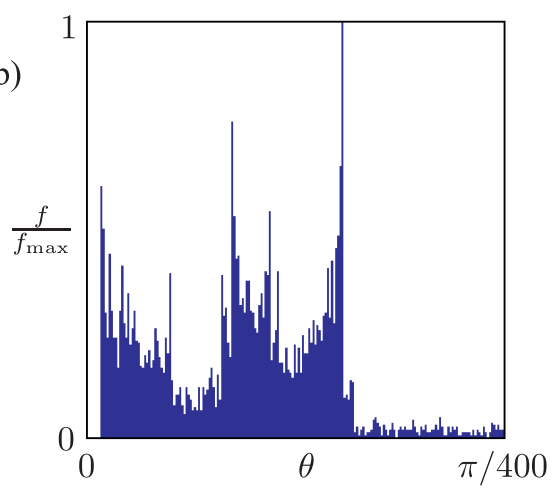

Fig. 4. (a) Chart of regimes of the system (4.1) on the plane $(\varepsilon, D)$, where areas of chaos and of periodic motions are shown. The region of hyperbolic chaos associated with the Smale-Williams attractor is shown in gray and marked as SW, other chaotic regimes are shown in white. The legend for periodic regimes is shown on the right. The periods indicated in colors are measured in units of the modulation period. Fixed parameters are $a=0.36, b=0.16, \mu=0.087, T=250, T_{0}=T / 4, \tau=T / 2$. (b) Histogram of the angles of intersection of stable and unstable subspaces for the hyperbolic attractor of the Poincaré map of the system (4.1). $a=0.36, b=0.16, \mu=0.087, \varepsilon=0.0003, D=0.8, T=250, T_{0}=T / 4$, $\tau=T / 2$.

\section{Conclusion}

We have discussed two mechanical models based on a Froude pendulum with SmaleWilliams hyperbolic attractors. Both models were simulated numerically. Areas of hyperbolic dynamics were identified in the parameter space checking the topological nature of the map for the angle variable. The hyperbolicity of the chaotic attractors was also confirmed using a criterion based on the analysis of the intersection angles of stable and unstable invariant subspaces of small perturbation vectors and checking the absence of tangencies between these subspaces.

\section{References}

[1] Shilnikov, L., Mathematical Problems of Nonlinear Dynamics: A Tutorial, Internat. J. Bifur. Chaos Appl. Sci. Engrg., 1997, vol. 7, no. 9, pp. 1953-2001.

[2] Smale, S., Differentiable Dynamical Systems, Bull. Amer. Math. Soc., 1967, vol. 73, no. 6, pp. 747-817.

[3] Williams, R., Expanding Attractors, Publ. Math. Inst. Hautes Études Sci., 1974, vol. 43, pp. 169-203.

[4] Kuznetsov, S. P. and Kruglov, V. P., Hyperbolic Chaos in a System of Two Froude Pendulums with Alternating Periodic Braking, Commun. Nonlinear Sci. Numer. Simul., 2019, vol.67, pp. 152-161.

[5] Kuznetsov, S. P. and Sedova, Yu. V., Robust Hyperbolic Chaos in Froude Pendulum with Delayed Feedback and Periodic Braking, Internat. J. Bifur. Chaos Appl. Sci. Engrg., 2019, vol.29, no.12, 1930035.

[6] Kuptsov, P. V., Fast Numerical Test of Hyperbolic Chaos, Phys. Rev. E, 2012, vol. 85, no. 1, 015203, $4 \mathrm{pp}$.

[7] Kuptsov, P. V. and Kuznetsov, S. P., Numerical Test for Hyperbolicity of Chaotic Dynamics in TimeDelay Systems, Phys. Rev. E, 2016, vol.94, no. 1, 010201, 6 pp. 